\title{
Fuerza prensil como factor predictor de Ansiedad-Rasgo en estudiantes universitarias
}

\author{
Grip strength as a predictor of Trait-Anxiety in university students \\ Álvaro Huerta-Ojeda1* orcid.org/0000-0001-6871-098X \\ Guillermo Barahona-Fuentes ${ }^{1}$ orcid.org/0000-0003-4913-9321 \\ Sergio Galdames-Maliqueo² orcid.org/0000-0002-0261-5741 \\ Pablo Cáceres-Serrano3 ${ }^{3}$ orcid.org/0000-0002-1691-9199 \\ Nelson Castillo-Hernández ${ }^{2}$ orcid.org/0000-0001-6284-6819 \\ Paula Ortiz-Marholz ${ }^{4}$ orcid.org/0000-0003-2535-8642
}

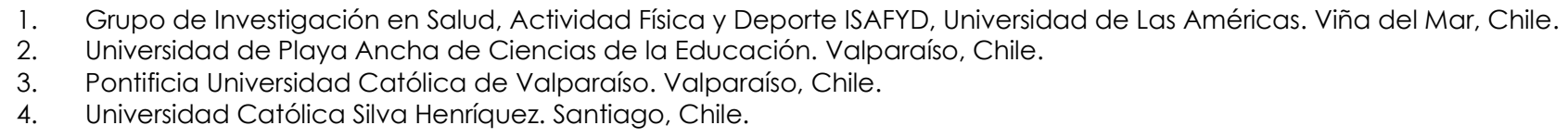

Fecha de recepción: Mayo 19 - $2020 \quad$ Fecha de revisión: Noviembre 25 - $2020 \quad$ Fecha de aceptación: Abril 29 - 2021

Huerta-Ojeda AC, Barahona-Fuentes G, Galdames-Maliqueo S, Cáceres-Serrano P, Castillo-Hernández N, Ortiz-Marholz P. Fuerza prensil como factor predictor de Ansiedad-Rasgo en estudiantes universitarias. Univ. Salud. 2021;23(2):100-108. DOI: https://doi.org/10.22267/rus.212302.221

\section{Resumen}

Introducción: Investigaciones han determinado que la implementación de programas de actividad física mejora la salud mental. Sin embargo, aún existen dudas sobre la relación entre fuerza muscular y la presencia de trastornos psicosociales como ansiedad y estrés en la población universitaria. Objetivo: Relacionar la condición física, específicamente la fuerza prensil, con la presencia de Ansiedad-Rasgo en estudiantes mujeres. Materiales y métodos: Investigación empírica con estrategia asociativa predictiva, con un diseño predictivo transversal. La muestra fue de 30 estudiantes mujeres de pregrado. Las variables físicas predictoras evaluadas fueron antropometría, fuerza prensil y consumo máximo de oxígeno, mientras que la Ansiedad-Rasgo se midió por medio del Cuestionario de Ansiedad Estado Rasgo (STAI). Se realizó análisis estadístico con una regresión logística ordinal entre las variables físicas predictoras y nivel de Ansiedad-Rasgo. Resultados: Se evidenció que sólo la fuerza prensil fue estadísticamente significativa $(p=0,033)$. Este análisis expresado en Odds Ratio, asume un 74,36\% de probabilidad que a un menor nivel de fuerza prensil, mayor presencia de Ansiedad-Rasgo. Conclusiones: La fuerza prensil, además de ser un parámetro físico que permite detectar riesgo cardiovascular y patologías asociadas, también debería ser considerada en la determinación de alteraciones en salud mental.

Palabras clave: Fuerza muscular; ansiedad; mujeres. (Fuente: DeCS, Bireme).

\begin{abstract}
Introduction: Research has revealed that the implementation of physical activity programs improves mental health. However, there are still doubts about the relationship between muscle strength and the presence of psychosocial disorders such as anxiety and stress in the university student population. Objective: To study the association of a physical condition (grip strength) and the presence of Trait-Anxiety in female students. Materials and methods: An empirical research with a predictive associative strategy was conducted on a sample of 30 female undergraduate students. The predictor physical variables assessed were anthropometry, grip strength, and maximum oxygen consumption. Trait-Anxiety was evaluated using the State-Trait Anxiety Inventory (STAI). Statistical analysis was performed through ordinal logistic regression between predictor physical variables and level of Trait-Anxiety. Results: Only grip strength was statistically significant ( $p=0.033$ ). This analysis, expressed as Odds Ratio, assumes a 74.36\% probability that the lower level of grip strength, the greater chances of having Trait-Anxiety. Conclusions: Grip strength is not only a physical parameter to detect cardiovascular risks and associated pathologies, but also it should be used to identify mental health disorders.
\end{abstract}

Key words: Muscle strength; anxiety; female. (Source: DeCS, Bireme).

\footnotetext{
*Autor de correspondencia

Álvaro Cristian Huerta Ojeda

e-mail: achuertao@yahoo.es
} 


\section{Introducción}

En Chile, casi un tercio de la población ha tenido alteraciones en su salud mental durante su vida, y el $22,2 \%$ en el último año, siendo la ansiedad junto a la depresión los trastornos psicosociales más prevalentes $^{(1)}$. En términos generales, alteraciones negativas en la salud mental desencadenan un deterioro en la calidad de vida de las personas que las padecen, generando falta de vitalidad, problemas emocionales, alteraciones en el trabajo y en las actividades diarias(2). La ansiedad como trastorno psicosocial, es considerada como un estado psicoemocional negativo donde se pueden observar y manifestar sensaciones en las que predomina la preocupación y el nerviosismo, y se refleja en componentes somáticos y cognitivos ${ }^{(3,4)}$.

La literatura describe dos tipos de ansiedades no clínicas, la primera de ellas hace referencia al rasgo individual que predispone a responder de forma nerviosa ante estímulos y situaciones de la vida cotidiana que la persona interpreta como amenazantes, y se conoce como "Ansiedad-Rasgo" (A-R); mientras que el segundo tipo se denomina "Ansiedad-Estado" (A-E), y se caracteriza por manifestaciones de ansiedad que se producen de forma breve en el tiempo, la mayoría de las veces, al enfrentar estresores reales(5).

De forma específica, y al igual que los otros grupos etarios, la población universitaria también puede padecer alteraciones en la salud mental(6), experimentando ansiedad en alguna etapa de su carrera; estos trastornos psicosociales se caracterizan por una sensación de aprensión difusa, desagradable y vaga, que se manifiesta por la incapacidad de estar sentado o de pie durante un tiempo, y a menudo se acompaña de síntomas vegetativos como cefalea, diaforesis, taquicardia, opresión torácica, malestar epigástrico e inquietud(7). Una de las primeras medidas que ayudan a disminuir estos trastornos es la detección temprana, por tal razón, se han desarrollado y adaptado test psicológico de A-E o A-R que buscan entregar herramientas a los profesionales para pesquisar aquellos estudiantes con alteraciones en la salud mental(5).

Esta detección temprana de alteraciones en la salud mental de los estudiantes permitiría, entre otras alternativas, que los equipos multidisciplinarios pudieran aplicar medidas paliativas y preventivas, no sólo medicamentosas y terapéuticas, sino que también implementar, por ejemplo, métodos de entrenamiento y/o actividad física que sean capaces de ayudar en el control y disminución de los trastornos psicosociales en las distintas poblaciones $(6)$.

En relación a esto último, existen algunas investigaciones que han utilizado métodos de entrenamiento físico para la reducción de los niveles de ansiedad y estrés(8). Un ejemplo de ello, fueron los ejercicios individuales de atención y relajación implementados por Gallego et al.,(9) durante una clase de educación física; al parecer estas actividades ayudarían a reducir las manifestaciones de estrés y ansiedad causadas por los exámenes en los estudiantes. La investigación de Gallego et al.,(9) junto a otras, han permitido evidenciar que el ejercicio puede producir una pequeña reducción en la ansiedad, sin embargo las investigaciones aún están sesgadas por la heterogeneidad de las muestras, ya que el tipo y la cantidad de personas inmersas en los estudios no permiten generalizar sus resultados(10).

Paralelamente, en los últimos años la evaluación y desarrollo de la fuerza ha tomado un rol clave en la génesis del metabolismo muscular alterado $\mathrm{y}$, por lo tanto, en la prevención de enfermedades patológicas comunes y enfermedades crónicas(11). También, se ha evidenciado que durante la infancia y la adolescencia, el nivel muscular se relaciona de forma inversa con factores de riesgo cardiovascular(12), y que en la edad adulta la masa muscular y la fuerza disminuyen paulatinamente(13). Esta amplia evidencia del desarrollo de la fuerza asociada a disminución del riesgo cardio-metabólico y calidad de vida, no da espacios para dudas(6,13). Sin embargo, y basado en el hecho que la actividad física podría ayudar a reducir las manifestaciones de estrés(9), quizás una buena condición física influya positivamente en la salud mental de la población.

Desafortunadamente, la relación entre una buena condición física general, específicamente niveles elevados de fuerza muscular, y la presencia de trastornos psicosociales como la A-R no está del todo esclarecida(10).

Por lo anterior y considerando que varias investigaciones han determinado que la implementación de programas de actividad física mejora la salud mental(8,14), y además que mayores 
niveles de fuerza muscular han sido asociados a un menor riesgo cardiovascular y enfermedades crónicas(12), el propósito principal de este estudio fue relacionar la condición física, específicamente la fuerza prensil, con la presencia de A-R en mujeres estudiantes de la Universidad de Las Américas de Chile.

\section{Materiales y métodos}

\section{Diseño de investigación}

Investigación empírica con estrategia asociativa predictiva, con un diseño predictivo transversal(15).

\section{Procedimientos}

Se convocó a todas las estudiantes mujeres que formaba parte de las actividades curriculares de libre elección, impartidas por la Universidad de Las Américas de Chile. Las estudiantes aceptaron voluntariamente ser parte del estudio (muestra no probabilística). Antes de comenzar las evaluaciones, se informó a las participantes el propósito y procedimientos del estudio, entre ellos el Cuestionario de A-R y las pruebas campo.

El criterio de inclusión fue que todas las estudiantes debían estar matriculadas en alguna carrera de pregrado en la Universidad, mientras que los criterios de exclusión fueron la prevalencia de lesiones músculo-esqueléticas, patologías cardíacas preexistente y la incapacidad de ejecutar las pruebas de campo. A todas las participantes se les solicitó que 48 horas previas a las mediciones no realizaran actividades físicas que generaran fatiga nerviosa $o$ músculo-esqueléticas, y que se abstuvieran de ingerir cafeína o cualquier sustancia que pudiera aumentar su metabolismo durante la evaluación.

Solamente aquellas participantes que firmaron el consentimiento informado fueron sometidas al Cuestionario de A-R y las pruebas de campo.

\section{Participantes}

Treinta estudiantes mujeres de pregrado, que formaban parte de las actividades curriculares de libre elección impartidas por la Universidad de Las Américas de Chile, se ofrecieron como voluntarias para participar en este estudio (Tabla 1). El tipo de muestreo fue no probabilístico por conveniencia. Semestralmente, son 120 estudiantes mujeres de pregrado que participan en las actividades curriculares de libre elección impartidas por la
Universidad de Las Américas, por la tanto, este estudio abarcó al $25 \%$ de la población total.

\section{Mediciones antropométricas}

La estatura $(\mathrm{cm})$ fue evaluada a través de un estadiómetro desde los pies hasta el vertex (plano de Frankford). El peso (Kg) y el porcentaje graso (\%) fueron evaluados a través de una balanza digital Tanita Inner Scan BC-554 ${ }^{\circledR}$. El peso, la estatura y el porcentaje graso se evaluaron con las estudiantes descalzas, pantalón corto y una polera ligera. La interpretación del Índice de Masa Corporal (IMC) se realizó según los estándares antropométricos para evaluación del estado nutritivo(16). Mientras que el perímetro de cintura (PC) se midió $2 \mathrm{~cm}$ sobre el ombligo con una cinta métrica metálica F.A.G.A. ${ }^{\circledR}$. Por último, la relación cintura estura (RCE) se obtuvo dividiendo el peso corporal $(\mathrm{cm})$ con la estatura (cm).

\section{Cuestionario de Ansiedad Estado-Rasgo STAI (State-Trait Anxiety Inventory)}

Este cuestionario permite investigar los fenómenos de la ansiedad, y consta de dos escalas: A-R y A-E, con 20 elementos cada una. Las 40 preguntas del cuestionario son valoradas mediante una escala Likert, abarcando cuatro opciones: $0=$ casi nunca, $1=\mathrm{a}$ veces, $2=a$ menudo, $3=$ casi siempre. La primera escala, de A-R, permite describir cómo se siente el participante en un "momento particular"; señala una relativamente estable propensión ansiosa donde se observa la tendencia a percibir las situaciones como amenazadoras y a elevar, consecuentemente, su A-E.

No son necesariamente personas que se muestren ansiosas constantemente, ya que puede ser que esa disposición permanezca latente hasta que es activada por algunos estímulos y que probablemente son disposiciones comportamentales adquiridas, es decir, residuos de experiencias pasadas que predisponen tanto a ver el mundo de una determinada manera como a manifestar tendencias de respuestas ansiosas ${ }^{(5)}$. Esta parte del cuestionario incluye siete preguntas de puntuación inversa (preguntas: 21, 26, 27, 30, 33, 36 y 39), mientras que las 13 restantes corresponden a preguntas de puntuación directa (preguntas: 22, 23, 24, 25, 28, 29 , $31,32,34,35,37,38$ y 40). Tanto las preguntas con puntuación directa como con puntuación inversa permiten definir los distintos niveles de A-R; para esto, se debe usar la siguiente ecuación(5): 


$$
\begin{aligned}
& A-R \\
& =21+\sum \text { preguntas } 21,26,27,30,33,36,39 \\
& -\sum \text { preguntas } 22,23,24,25,28,29,31,32,34,35,37,40
\end{aligned}
$$

La segunda escala, de A-E, permite describir un estado o condición emocional transitoria del organismo humano, que se caracteriza por sentimientos subjetivos, conscientemente percibidos, de tensión y aprehensión, así como por una hiperactividad del sistema nervioso autónomo que puede variar en el tiempo y fluctuar en intensidad(5). Esta parte del cuestionario incluye 10 preguntas de puntuación inversa (preguntas: 3, 4, 6, $7,9,12,13,14,17,18)$, mientras que las 10 restantes corresponden a preguntas de puntuación directa (preguntas: 1, 2, 5, 8, 10, 11, 15, 16, 19 y 20). Tanto las preguntas con puntuación directa como con puntuación inversa permiten definir los distintos niveles de A-E; para esto, se debe usar la siguiente ecuación(5):

$$
\begin{aligned}
& A-E=21+\sum \text { preguntas } 1,2,5,8,10,11,15,16,19,20 \\
&-\sum \text { preguntas } 3,4,6,7,9,12,13,14,17,18
\end{aligned}
$$

Al aplicar la fórmula para determinar A-R $\mathrm{y}$ dependiendo del puntaje obtenido, los sujetos evaluados se clasifican en cinco niveles: de 0 a 16 puntos: "bajo", de 17 a 23 puntos: "tendiente al promedio", de 24 a 25 puntos: "promedio", de 26 a 32 puntos "sobre el promedio", y de 33 a 60 puntos: "alto". Al aplicar la fórmula para determinar A-E y dependiendo del puntaje obtenido, los sujetos evaluados se clasifican en cinco niveles: de 0 a 14 puntos: "bajo", de 15 a 19 puntos: "tendiente al promedio", de 20 a 22 puntos: "promedio", de 23 a 31 puntos: "sobre el promedio", y de 32 a 60 puntos: "alto".

El coeficiente de fiabilidad para los ítems que miden A-E en este estudio fueron los siguientes: preguntas con puntuación directa 0,86 y preguntas con puntuación inversa 0,88 en el coeficiente de Alpha de Cronbach, respectivamente. Paralelamente, el coeficiente de fiabilidad para los ítems que miden A$\mathrm{R}$ fueron los siguientes: preguntas con puntuación directa 0,74 y preguntas con puntuación inversa 0,77 en el coeficiente de Alpha de Cronbach, respectivamente.

\section{Fuerza prensil}

Esta prueba permite evaluar la prensión máxima de las manos en $\mathrm{Kg}^{(17)}$. Antes de su aplicación, el dinamómetro se ajustó al tamaño de la mano. La evaluación fue en posición de pie, con el brazo derecho al costado del cuerpo, el codo extendido y manteniendo la fuerza prensil máxima por tres segundos. Para la evaluación de la fuerza prensil, se utilizó un dinamómetro manual modelo Smedley ${ }^{\circledR} \mathrm{de}$ 100 Kg (TTM, Tokio).

\section{Test de Astrand}

El objetivo de este test submaximal es determinar la potencia aeróbica máxima $\left(\mathrm{VO}_{2 \text { máx }}\right)$ en sujetos de baja aptitud física. Para el desarrollo de este test en mujeres, se necesita un metrónomo (90 batidas por minuto) y un escalón de $33 \mathrm{~cm}$. Las participantes subieron al escalón 22,5 veces por minuto durante 5 minutos. Para evaluar la Frecuencia Cardiaca (FC) de término se utilizó un monitor de ritmo cardíaco marca Polar Team $®$, y para la determinación del $\mathrm{VO}_{2 \text { máx }}$ se empleó el nomograma descrito para el test(18).

\section{Análisis estadístico}

Para el análisis de datos, las variables físicas predictoras (antropometría, $\mathrm{VO}_{2 \text { máx }}$ y de fuerza prensil) y A-R fueron sometidas al test de normalidad Shapiro-Wilk. Luego, se efectuó un análisis de regresión logística ordinal de variables físicas predictoras sobre nivel de A-R(19). Los análisis descriptivos y de correlación fueron realizados mediante el entorno de programación estadística $\mathrm{R}^{(20)}$. El nivel de significancia para todos los análisis estadísticos fue de $p<0,05$.

\section{Consideraciones éticas}

El consentimiento informado y el estudio fueron aprobados por el Comité de Investigación Humana de la Universidad de Las Américas, Chile (registro número PI-022-2018) y se llevó a cabo de conformidad con la Declaración de Helsinki (WMA 2000, Bošnjak 2001, Tyebkhan 2003) que establece los principios éticos fundamentales para la investigación con seres humanos.

\section{Resultados}

En la Tabla 1 se describen algunas variables antropométricas de las participantes del estudio.

Al término de la aplicación del Cuestionario de Ansiedad Estado-Rasgo, los 30 casos fueron estratificados según los niveles de A-R de la siguiente manera: "Bajo" $(\mathrm{n}=8 ; 26,6 \%)$, "Tendiente al promedio" (n=9; 30\%), "Promedio" (n=3; 10\%), 
"Sobre el promedio" (n=5; 16,7\%), y "Alto" (n=5; 16,7\%) (Tabla 2).

Al realizar el análisis de regresión ordinal (modelo completo) con todas las variables físicas predictoras, se observó que sólo la fuerza prensil fue estadísticamente significativa (Tabla 3).
Tabla 1. Características de los participantes

\begin{tabular}{|c|c|}
\hline & Media \pm DS (mín - máx) \\
\hline Edad (años) & $24,6 \pm 5,2(20-46)$ \\
\hline Peso (Kg) & $63,0 \pm 11,2(44,4-87,3)$ \\
\hline Estatura $(\mathrm{cm})$ & $156,4 \pm 5,3(145-167)$ \\
\hline $\mathrm{IMC}\left(\mathrm{Kg} / \mathrm{m}^{2}\right)$ & $25,7 \pm 4,5(18,5-36,3)$ \\
\hline Perímetro de cintura $(\mathrm{cm})$ & $74,1 \pm 13,5(51-110)$ \\
\hline RCE & $0,47 \pm 0,08(0,31-0,73)$ \\
\hline$\%$ Grasa & $30,8 \pm 7,6(15,7-43,7)$ \\
\hline $\mathrm{VO}_{2 \text { máx }}\left(\mathrm{mlO}_{2} \cdot \mathrm{Kg}^{-1} \cdot \mathrm{min}^{-1}\right)$ & $32,6 \pm 5,5(20,9-44,3)$ \\
\hline Fuerza prensil (Kg) & $25,0 \pm 3,8(17,0-31,5)$ \\
\hline
\end{tabular}

Tabla 2. Descripción de variables según niveles de $A-R(n=30)$

\begin{tabular}{|c|c|c|c|c|c|}
\hline & $\begin{array}{c}\text { Bajo } \\
(n=8 ; 26,6 \%)\end{array}$ & $\begin{array}{c}\text { Tendiente al } \\
\text { promedio } \\
(\mathrm{n}=9 ; 30 \%)\end{array}$ & $\begin{array}{c}\text { Promedio } \\
(n=3 ; 10 \%)\end{array}$ & $\begin{array}{l}\text { Sobre el promedio } \\
\quad(n=5 ; 16,7 \%)\end{array}$ & $\begin{array}{c}\text { Alto } \\
(n=5 ; 16,7 \%)\end{array}$ \\
\hline Centil A-R & $6,2 \pm 4,9(1-15)$ & $37,2 \pm 8,3(25-45)$ & $60,0 \pm 0,0(60-60)$ & $74,0 \pm 7,4(65-85)$ & $83,0 \pm 2,7(80-85)$ \\
\hline Edad (años) & $25,0 \pm 3,8(21-30)$ & $24,3 \pm 3,0(21-31)$ & $24 \pm 3,6(20-27)$ & $21 \pm 1,7(20-24)$ & $28,8 \pm 10,1(21-46)$ \\
\hline Peso (Kg) & $67,9 \pm 9,2(54,5-81,6)$ & $60,3 \pm 13,0(47,6-87,3)$ & $61,5 \pm 9,3(54,6-72,1)$ & $58,3 \pm 8,4(44,4-66,7)$ & $66 \pm 14,4(50,6-84)$ \\
\hline Estatura $(\mathrm{cm})$ & $157,5 \pm 6,2(145-167)$ & $157 \pm 2,5(154-160)$ & $156 \pm 4(152-160)$ & $155,4 \pm 6,6(149-164)$ & $155 \pm 8,3(147-167)$ \\
\hline $\mathrm{IMC}\left(\mathrm{Kg} / \mathrm{m}^{2}\right)$ & $27,4 \pm 3,5(21-32,1)$ & $24,5 \pm 5,4(18,5-36,3)$ & $25,2 \pm 2,8(22,4-28,1)$ & $24,1 \pm 3,3(19,9-28,4)$ & $27,4 \pm 5,7(22,7-36,3)$ \\
\hline $\begin{array}{l}\text { Perímetro de } \\
\text { cintura }(\mathrm{cm})\end{array}$ & $75,8 \pm 8,4(61,5-91)$ & $73,6 \pm 16,3(51-110)$ & $69,6 \pm 6,6(64-77)$ & $70,6 \pm 12,4(58-89)$ & $78,6 \pm 20,6(59-108)$ \\
\hline RCE & $0,48 \pm 0,05(0,38-0,54)$ & $0,46 \pm 0,10(0,31-0,70)$ & $0,44 \pm 0,05(0,41-0,50)$ & $0,45 \pm 0,07(0,36-0,55)$ & $0,50 \pm 0,14(0,36-0,73)$ \\
\hline$\%$ Grasa & $34,5 \pm 4,7(23,9-39,7)$ & $28,8 \pm 8,0(16,6-43,7)$ & $30,4 \pm 6,4(25,7-37,8)$ & $27,6 \pm 8,7(15,7-36,2)$ & $32 \pm 10,3(20,5-43,6)$ \\
\hline $\begin{array}{l}\mathrm{VO}_{2 \max }\left(\mathrm{mlO}_{2} \cdot \mathrm{Kg}\right. \\
\left.{ }^{-} \cdot \mathrm{min}^{-1}\right)\end{array}$ & $34,5 \pm 5,9(29,6-44,3)$ & $30,2 \pm 3,7(20,9-33,6)$ & $35,6 \pm 6,7(30,5-43,2)$ & $30,3 \pm 3,2(28,4-36)$ & $34,3 \pm 7,7(23,7-42,9)$ \\
\hline $\begin{array}{l}\text { Fuerza prensil } \\
(\mathrm{Kg})\end{array}$ & $28,2 \pm 2,5(23,8-31,2)$ & $23,8 \pm 4,4(19,3-31,5)$ & $24,7 \pm 2,0(23,2-27,0)$ & $24,0 \pm 2,6(20,9-27,5)$ & $23,4 \pm 4,1(17,0-26,9)$ \\
\hline
\end{tabular}

Como se aprecia en los coeficientes del modelo (Tabla 4), sólo la fuerza prensil fue estadísticamente significativa $(0,033)$, mientras que el análisis expresado en odds ratio (OR), que representa la magnitud de asociación entre dos variables, asume un $74,36 \%$ de probabilidad que a un menor nivel de fuerza prensil, mayor nivel de A-R. A su vez, para hacer una segunda interpretación se puede calcular el inverso $(100 / 74,36=1,24)$; esto significa que un sujeto con menor nivel de fuerza prensil tiene 1,24 veces más probabilidades de tener un nivel "sobre el promedio" o "altos" de A-R.
Tabla 3. Test de razón de verosimilitud general

\begin{tabular}{lrrr}
\hline \multicolumn{1}{c}{ Predictor } & \multicolumn{1}{c}{$\mathbf{x}^{\mathbf{2}}$} & df & p \\
\hline $\mathrm{IMC}\left(\mathrm{Kg} / \mathrm{m}^{2}\right)$ & 1,706 & 1 & 0,192 \\
$\mathrm{RCE}$ & 0,385 & 1 & 0,535 \\
$\%$ Grasa & 0,747 & 1 & 0,387 \\
$\mathrm{VO}_{2 \text { máx }}\left(\mathrm{mlO}_{2} \cdot \mathrm{Kg}^{-1} \cdot \mathrm{min}^{-1}\right)$ & $4,51 \mathrm{e}-4$ & 1 & 0,983 \\
Fuerza prensil $(\mathrm{Kg})$ & 5,011 & 1 & 0,025
\end{tabular}

A-R (ansiedad rasgo); IMC (índice de masa corporal); RCE (relación cintura-estatura); $\mathrm{VO}_{2 \text { máx }}$ (consumo máximo de oxígeno); $\mathrm{mlO}_{2} \cdot \mathrm{Kg}^{-1} \cdot \mathrm{min}^{-1}$ (mililitros de oxígeno por kilogramo de peso corporal por minuto). 
Tabla 4. Coeficientes del modelo

\begin{tabular}{lccccccccc}
\hline \multicolumn{1}{c}{ Predictor } & Estimación & Bajo* $^{*}$ & Alto* & EE & $\mathbf{Z}$ & P & Odds ratio & Bajo* & Alto* \\
\hline IMC & 0,32954 & $-0,1613$ & 0,8663 & 0,2588 & 1,2731 & 0,203 & 1,39032 & 0,8510 & 2,378 \\
$\mathrm{RCE}$ & $-5,75543$ & $-24,7980$ & 12,3723 & 9,3479 & $-0,6157$ & 0,538 & 0,00317 & $1,70 \mathrm{e}-11$ & 236172 \\
\% Grasa & $-0,09031$ & $-0,3058$ & 0,1133 & 0,1055 & $-0,8557$ & 0,392 & 0,91365 & 0,7365 & 644 \\
$\mathrm{VO}_{2 \text { máx }}\left(\mathrm{mlO}_{2} \cdot \mathrm{Kg}^{-1} \cdot \mathrm{min}^{-1}\right)$ & $-0,02277$ & $-2,5382$ & 2,4117 & 1,2423 & $-0,0183$ & 0,985 & 0,97748 & 0,0790 & 1,120 \\
Fuerza prensil & $-0,29623$ & $-0,5942$ & $-0,0363$ & 0,1386 & $-2,1371$ & 0,033 & 0,74361 & 0,5520 & 0,964 \\
\hline
\end{tabular}

*Intervalo de confianza del 95\%

IMC (índice de masa corporal); RCE (relación cintura-estatura); $\mathrm{VO}_{2 \text { máx }}$ (consumo máximo de oxígeno); $\mathrm{mlO} \cdot \mathrm{Kg}^{-1} \cdot \mathrm{min}^{-1}$ (mililitros de oxígeno por kilogramo de peso corporal por minuto).

\section{Discusión}

En relación al objetivo principal del estudio, al término del análisis, se observó una nula relación entre las variables antropométricas y el $\mathrm{VO}_{2 \text { máx }}$ con los niveles de A-R, sin embargo, la fuerza prensil demostró ser un buen predictor de A-R en estudiantes universitarias. Los antecedentes presentados en esta investigación toman relevancia cuando se evidencia que los problemas de salud mental (ansiedad y estrés), afectan a todo tipo de sujetos sin excepción alguna(21). Es así como, la población universitaria no está ajena a estos problemas de salud mental, ya que el padecimiento de trastornos psicosociales como la ansiedad y el estrés se han asociado a la baja de rendimiento académico(6). Lo anterior, ha desencadenado que las instituciones universitarias consideren estos trastornos psicosociales como una de las preocupaciones más trascendentales en relación a la salud mental del alumnado(22).

En Chile, la realidad no es distinta, debido a que los estudiantes universitarios constituyen uno de los grupos objetivos de la Política Nacional de Promoción de Salud(23). Este contexto ha motivado el desarrollo de guías y programas que ayuden al control y/o disminución de los trastornos psicosociales como la ansiedad y el estrés(24). Dichos métodos han sido tratamientos medicamentosos(25), terapias alternativas(26) 0 ejercicio físico(8).

De manera específica, en el presente estudio sólo la fuerza prensil fue estadísticamente significativa $(0,033)$ al ser correlacionada con la ansiedad, demostrando que las mujeres universitarias con un mayor nivel de fuerza muscular poseen 1,24 veces menos posibilidad de padecer A-R (Tabla 4). En este sentido, Németh et al.,(27) investigaron los cambios en los parámetros psicofisiológicos provocados por el estrés físico en ejercicios intensos de corta duración en estudiantes universitarios; el análisis indicó correlaciones significativas entre la fuerza muscular (fuerza prensil) y el estrés cardíaco, tanto para estudiantes de medicina $(r=-0,648, p<0,031)$ como para estudiantes que practicaban deporte $(r=-$ $0,682, p<0,030$ ); en ambos casos se concluyó que cuanto mayor es la fuerza muscular de los sujetos, menor es el nivel de estrés, influyendo de manera ventajosa en la salud mental de los participantes.

Paralelamente, los niveles absolutos de fuerza prensil evaluados en el presente estudio fueron de $25,0 \pm 3,8 \mathrm{Kg}$, valor levemente más bajo que los parámetros internacionales aceptados (mano derecha 30,0 \pm 7,0 Kg; mano izquierda $28 \pm 6,1$ $\mathrm{Kg})^{(28) .}$ Quizás este antecedente (niveles absolutos de fuerza prensil) sea el más relevante del estudio, al demostrar que 22 de las 30 mujeres universitarias evaluadas estuvieron dentro de los rangos "Tendiente al promedio", "Promedio", "Sobre el promedio" y "Alto" en el Cuestionario de Ansiedad Estado-Rasgo(5).

De igual forma, en otra investigación que tuvo por objetivo comparar las respuestas psicobiológicas y percepcionales agudas en hombres normotensos e hipertensos, se concluyó que el entrenamiento de fuerza manual parece ser una alternativa viable para mejorar las respuestas psicobiológicas agudas(29). A su vez, en un meta análisis desarrollado por Ensari et al.,(10) se concluyó que la evidencia acumulada de estudios de alta calidad convergen en que los ejercicios agudos pueden producir una reducción en la ansiedad.

En una investigación desarrollada por Gorham et al.,(14) se explica que la reducción de los trastornos psicosociales, asociada a la participación deportiva, puede ser relacionada a un mecanismo neural, ya que el ejercicio posee un impacto en el desarrollo del cerebro, específicamente el ejercicio físico 
provocaría un aumento en el volumen del hipocampo. Sin embargo, los mismos autores mencionan que se necesitan más investigaciones para comprender las relaciones causales entre estas variables(14).

Consecuentemente, al parecer la fuerza prensil es un parámetro físico que debería ser considerado para determinar alteraciones en la salud mental de la población, por esta misma razón, sería interesante analizar las variaciones en los niveles de A-R y A-E posterior a un programa de ejercicio físico orientado al desarrollo de la fuerza muscular(30).

En el presente estudio, el $\mathrm{VO}_{2 \text { máx }}$ no mostró ser un factor predictor de la ansiedad $(p=0,983)$ (Tabla 3$)$. Sin embargo, en un estudio realizado por Candel et al.,(31) se menciona que la práctica de algún tipo de actividad física tienen puntuaciones superiores en todos los factores de autoconcepto en mujeres, excepto en el emocional. Es así como diversas investigaciones han desarrollado distintos métodos de entrenamiento a través de la actividad física, el ejercicio físico y el deporte, para producir algún tipo de cambio sustancial en los parámetros de salud mental, entre estos métodos, se encuentran programas de baile, definidos dentro de la práctica de actividad física grupal(30,32), ejercicio físico moderado-vigoroso(33), técnicas de relajación(34), entre otros métodos de actividad física.

De forma más específica, Gerber et al.,(6) asociaron distintos tipos de ejercicio con las percepciones de estrés, los investigadores concluyeron que el ejercicio aeróbico no posee un efecto moderador de estrés, mientras que el levantamiento de pesas sólo se asoció con síntomas depresivos más bajos entre los estudiantes con bajo estrés percibido; estos hallazgos sugieren que ciertos ejercicios pueden tener un mejor potencial para moderar la relación entre el estrés percibido y los síntomas depresivos que otros.

Pese a estos antecedentes, algunas revisiones han demostrado que las personas con aptitud aeróbica se recuperaron más rápidamente del estrés relacionado con el trabajo(35), por lo que quizás la principal razón de la nula correlación entre el $\mathrm{VO}_{2 \text { máx }}$ y los niveles de ansiedad en estudiantes universitarias en este estudio fue un error de medición(18), ya que el protocolo utilizado posee un bajo nivel de confiabilidad, debido a que estima el $\mathrm{VO}_{2 \text { máx, y no lo }}$ mide directamente; por esta razón, sería interesante correlacionar nuevamente los niveles de A-R y A-E con un test directo de $\mathrm{VO}_{2 \text { máx }}$ en estudiantes universitarias(36).

Desde el punto de vista práctico, los profesionales que usen la fuerza prensil como un predictor de presencia de ansiedad en la población universitaria, deben considerar las respuestas individuales de los participantes, ya que los perfiles psicológicos presentes en cada uno de estos podría condicionar el estado de ansiedad(37). Además, para aplicar todas las pruebas físicas declaradas en este estudio, los profesionales deben resguardar la integridad de los pacientes, evitando la aplicación de estas pruebas en sujetos con enfermedades preexistentes o patologías que impidan una correcta ejecución.

A pesar de los resultados encontrados en la presente investigación, y considerando que este trabajo resulta pionero en relacionar la fuerza prensil con la A-R, también tiene limitaciones que se deberían intentar subsanar en futuros estudios. Por un lado, y considerando la muestra reducida de estudiantes, los resultados obtenidos no pueden generalizarse a estudiantes de otras universidades que poseen entornos psicosociales distintos.

\section{Conclusiones}

Los resultados de la presente investigación mostraron una nula relación entre las variables antropométricas y el $\mathrm{VO}_{2 \text { máx }}$ con los niveles de $\mathrm{A}-\mathrm{R}$, sin embargo, la fuerza prensil demostró ser un buen predictor de A-R en estudiantes universitarias, demostrando que los sujetos con mayor nivel de fuerza muscular poseen 1,24 veces menos posibilidad de padecer A-R.

Por lo anterior, la fuerza prensil es un parámetro físico que debería ser considerado para pesquisar no solamente riesgo cardiovascular y patologías asociadas, sino que también, parámetros de salud mental.

\section{Futuros estudios}

Durante la revisión literaria y el desarrollo de la presente investigación, se puedo evidenciar que en Chile, al menos en estudiantes universitarios, no se han aplicado protocolos de actividad física que tengan como propósito controlar los niveles de A-R. Por lo tanto, como una perspectiva de futuro, se espera implementar un protocolo de actividad física 
basado en ejercicios de fuerza para prevenir la aparición de A-R.

\section{Fuente de financiamiento}

Universidad de Las Américas, bajo los fondos concursables de Investigación N PI-022-2018.

Conflicto de intereses: Los autores no declaran conflicto de interés.

\section{Referencias}

1. Vicente B, Saldivia S, Pihán R. Prevalencias y brechas hoy; salud mental mañana. Acta Bioeth. 2016;22(1):51-61. Disponible en: https://doi.org/10.4067/s1726$569 \times 2016000100006$

2. Crempien C, de la Parra G, Grez M, Váldez C, López MJ, Krause M. Características sociodemográficas y clínicas de pacientes diagnosticados con depresión en Centros Comunitarios de Salud Mental (COSAM) de Santiago, Chile. Rev Chil Neuro-Psiquiatrica. 2017;55(1):26-35. Disponible en: https://doi.org/10.4067/s0717-92272017000100004

3. Kuan G, Morris T, Kueh YC, Terry PC. Effects of relaxing and arousing music during imagery training on dart-throwing performance, physiological arousal indices, and competitive state anxiety. Front Psychol. 2018;9(14):1-12. Disponible en: https://doi.org/10.3389/fpsyg.2018.00014

4. Castro-Sánchez M, Zurita-Ortega F, Chacón-Cuberos R, Lozano-Sánchez A. Clima motivacional y niveles de ansiedad en futbolistas de categorías inferiores. Retos. 2019;35:164$9 . \quad$ Disponible en: https://doi.org/10.47197/retos.v0i35.63308

5. Buela-Casal G, Guillén-Riquelme A, Seisdedos N. Cuestionario de Ansiedad Estado-Rasgo. In: TEA, editor. Cuest. Ansiedad Estado-Rasgo. 9a ed., Madrid, España: 2015; p. 1-39.

6. Gerber M, Brand S, Elliot C, Holsboer-Trachsler E, Pühse U. Aerobic Exercise, Ball Sports, Dancing, and Weight Lifting as Moderators of the Relationship between Stress and Depressive Symptoms: An Exploratory Cross-Sectional Study with Swiss University Students. Percept Mot Skills. 2014;119(3):679-97. Disponible en: https://doi.org/10.2466/06.PMS.119c26z4

7. Cardona-Arias JA, Pérez-Restrepo D, Rivera-Ocampos S, Gómez-Martínez J, Reyes Á. Prevalencia de ansiedad en estudiantes universitarios. Rev Divers - Perspect En Psicol. 2015;11(1):79-89. Disponible en: https://www.redalyc.org/articulo.oa?id=67943296005

8. Hernández B, Chávez E, Torres J, Torres A, Fleitas I. Evaluación de un programa de actividad físico-recreativa para el bienestar físico-mental del adulto mayor. Rev Cuba Investig Biomédicas. 2017;36(4):1-16. Disponible en: http://scielo.sld.cu/scielo.php?script=sci_arttext\&pid=S086 4-03002017000400007\&lng=e

9. Gallego J, Aguilar-Parra JM, Cangas AJ, Langer ÁI, Manãs I. Effect of a Mindfulness Program on Stress, Anxiety and Depression in University Students. Span J Psychol. 2014;17:e109. Disponible en: https://doi.org/10.1017/sjp.2014.102

10. Ensari I, Greenlee TA, Motl RW, Petruzzello SJ. MetaAnalysis of acute exercise effects on state anxiety: an update of randomized controlled trials over the past 25 years.
Depress Anxiety. 2015;32(8):624-34. Disponible en: https://doi.org/10.1002/da.22370

11. Mukund K, Subramaniam S. Skeletal muscle: A review of molecular structure and function, in health and disease. Rev Syst Biol Med. 2020;12(1):e1462. Disponible en: https://doi.org/10.1002/wsbm.1462

12. Fraser BJ, Huynh QL, Schmidt MD, Dwyer T, Venn AJ, Magnussen CG. Childhood Muscular Fitness Phenotypes and Adult Metabolic Syndrome. Med Sci Sports Exerc. 2016;48(9):1715-22. Disponible en: https://doi.org/10.1249/MSS.0000000000000955

13. Enríquez-Reyna MC, Bautista DC, Orocio RN. Nivel de actividad física, masa y fuerza muscular de mujeres mayores de la comunidad: Diferencias por grupo etario. Retos. 2019;35:121-5. Disponible en: https://doi.org/10.47197/retos.v0i35.59956

14. Gorham LS, Jernigan T, Hudziak J, Barch DM. Involvement in Sports, Hippocampal Volume, and Depressive Symptoms in Children. Biol Psychiatry Cogn Neurosci Neuroimaging. 2019;4(5):484-92. Disponible https://doi.org/10.1016/j.bpsc.2019.01.011

15. Ato M, López-García JJ, Benavente A. A classification system for research designs in psychology. An Psicol. 2013;29(3):1038-59. Disponible en: https://doi.org/10.6018/analesps.29.3.178511

16. Barrera MG. Estandáres antropométricos para evaluación del estado nutritivo. INTA. Santiago, Chile: 2004; Universidad de Chile.

17. Mathiowetz V. Comparison of Rolyan and Jamar dynamometers for measuring grip strength. Occup Ther Int. 2002;9(3):201-9. Disponible en: https://doi.org/10.1002/oti.165

18. Siconolfi SF, Cullinane EM, Carleton RA, Thompson PD. Assessing V02max in epidemiologic studies: modification of the Astrand-Rhyming test. Med Sci Sports Exerc. 1982;14(5):335-8. Disponible en: https://doi.org/10.1249/00005768-198205000-00004

19. Pallarés J, Rosel J, Jara MP, Herrero F, Calero MJ. Un ejemplo de la utilidad del modelo de regresión logística ordinal en estudios con variables de tipo frecuencial acumulativo utilizando el programa SPSS. In: Vasco U del P, editor. XII Congr. Metodol. las Ciencias Soc. y la Salud, Donostia, Espanya: 2011.

20. Revelle W. psych: Procedures for psychological, psychometric, and personality research. Software 2017.

21. Organización Mundial de la Salud. Depresión. OMS. 2020;13. Disponible en: https://www.who.int/es/newsroom/fact-sheets/detail/depression

22. McDonnell S, Semkovska M. Resilience as Mediator between Extraversion, Neuroticism, and Depressive Symptoms in University Students. J Posit Psychol Wellbeing. 2020;4(1):26-40. Disponible en: https://journalppw.com/index.php/JPPW/article/view/16 4

23. Salinas J, Vio F. Promoción de salud y actividad física en Chile: política prioritaria. Rev Panam Salud Pública. 2003;14(4):281-8. Disponible en: https://www.scielosp.org/pdf/rpsp/v14n4/18134.pdf

24. Lange I, Vio F. Guía para Universidades Saludables y otras Instituciones de Educación Superior. Santiago, Chile: 2006. Disponible http://www7.uc.cl/ucsaludable/img/guiaUSal.pdf

25. Ernst M, Lago T, Davis A, Grillon C. The effects of methylphenidate and propranolol on the interplay between 
induced-anxiety and working memory. Psychopharmacology (Berl). 2016;233(19):3565-74. Disponible en: https://doi.org/10.1007/s00213-016-4390$\mathrm{y}$

26. Herrera-Gutiérrez E, Olmos-Soria M, Brocal-Pérez D. Efectos psicológicos de la práctica del Método Pilates en una muestra universitaria. An Psicol. 2015;31(3):916-20. Disponible https://doi.org/10.6018/analesps.31.3.170101

27. Németh E, Bretz K, Sótonyi P, Bretz K, Horváth T, Tihanyi J, et al. Investigation of changes in psycho-physiological parameters evoked by short duration, intensive physical stress. Acta Physiol Hung. 2013;100(4):378-87. Disponible en:

https://akjournals.com/view/journals/036/100/4/articlep378.xml

28. Massy-Westropp NM, Gill TK, Taylor AW, Bohannon RW, Hill CL. Hand Grip Strength: age and gender stratified normative data in a population-based study. BMC Res Notes. 2011;4(127):1-5. Disponible en: https://doi.org/10.1186/1756-0500-4-127

29. Teixeira CVLS, Ferreira SE, Evangelista AL, da SilvaGrigoletto ME, Gomes RJ. Respostas psicobiológicas agudas do treinamento resistido com diferentes níveis de interação social. Rev Andaluza Med Del Deport. 2018;11(2):79-83. Disponible en: https://doi.org/10.1016/j.ramd.2015.11.004

30. Huerta Ojeda Á, Barahona-Fuentes G, Galdames-Maliqueo S, Cáceres-Serrano P, Ortiz-Marholz P. Efectos de un programa de Zumba ${ }^{\circledR}$ sobre niveles de ansiedad-rasgo, ansiedadestado y condición física en estudiantes universitarias chilenas. Cuad Psicol Del Deport. 2020;20(3):1-14. Disponible en: https://doi.org/10.6018/cpd.412371

31. Candel-Campillo N, Olmedilla-Zafra A, Blas-Redondo A. Relaciones entre la práctica del ejercicio físico y el autoconcepto, la ansiedad y la depresión en chicas adolescentes. Cuad Psicol Del Deport. 2008;8(1):61-77.
Disponible

en:

https://revistas.um.es/cpd/article/view/54541

32. Oberste $M$, Medele $M$, Javelle F, Wunram HL, Walter D, Bloch $\mathrm{W}$, et al. Physical Activity for the Treatment of Adolescent Depression: A Systematic Review and Meta-Analysis. Front Physiol. 2020;11:185. Disponible en: https://doi.org/10.3389/fphys.2020.00185

33. Liu M, Zhang J, Kamper-DeMarco KE, Hu E, Yao S. Associations of moderate-to-vigorous physical activity with psychological problems and suicidality in Chinese high school students: A cross-sectional study. PeerJ. 2020;8:e8775. Disponible en: https://doi.org/10.7717/peerj.8775

34. Neumark-Sztainer D, Wall MM, Choi J, Barr-Anderson DJ, Telke S, Mason SM. Exposure to Adverse Events and Associations with Stress Levels and the Practice of Yoga: Survey Findings from a Population-Based Study of Diverse Emerging Young Adults. J Altern Complement Med. 2020;26(6):482-90. Disponible en: https://doi.org/10.1089/acm.2020.0077

35. Forcier K, Stroud LR, Papandonatos GD, Hitsman B, Reiches M, Krishnamoorthy J, et al. Links between physical fitness and cardiovascular reactivity and recovery to psychological stressors: A meta-analysis. Heal Psychol. 2006;25(6):72339. Disponible en: https://doi.org/10.1037/02786133.25.6.723

36. Howley ET, Bassett DR, Welch H. Maximal oxygen uptake: "classical" versus"contemporary" viewpoints. Med Sci Sports Exerc. 1997;29(5):591-603. Disponible en: http://doi.org/10.1097/00005768-199705000-00002

37. Morillo-Baro PJ, Reigal-Garrido RE, Hernández-Mendo A. Relaciones entre el perfil psicológico deportivo y la ansiedad competitiva en jugadores de balonmano playa. Rev Psicol Del Deport. 2016;25(1):121-8. Disponible en: https://www.redalyc.org/articulo.oa?id=235143645016 